\title{
Performance of DSDV Protocol over Sensor Networks
}

\author{
Khushboo Tripathi, Tulika Agarwal and S. D. Dixit \\ Department of Electronics and Communications \\ University of Allahabad, Allahabad-211002, India
}

Khushboo83@live.com

Tulikabbs09@gmail.com

\begin{abstract}
Routing protocols play crucial role in determining performance parameters such as packet delivery fraction, end to end (end 2 end) delay, packet loss etc. of any ad hoc communication network. In this paper the performance of DSDV protocol in sensor network of randomly distributed static nodes with mobile source and sink nodes is investigated for source and sink velocities $0,10,20,40$ and $60 \mathrm{~m} / \mathrm{sec}$. and node densities $20-100$ nodes $/ \mathrm{km}^{2}$ by ns- 2 simulator. It is observed that the average simulation end to end delay in static source and sink nodes scenario (i.e. velocity $\mathrm{v}=0$ ) is generally higher than that in mobile source-sink cases for all node densities except at 20 nodes $/ \mathrm{km}^{2}$. In the case where source and sink nodes are mobile, the delay is almost constant at 0.12 seconds (except at $v 10=10 \mathrm{~m} / \mathrm{s}$ for 40 nodes $/ \mathrm{km}^{2}$ ). The average number of dropped packets in static case is higher than that in dynamic source-sink scenario. With increase in the velocities in case of dynamic source and sink nodes, the average number of dropped packets is around 10. The byte delivery fraction for moving source-sink cases for all velocities is higher than that for the static case (i.e. $v=0$ ) for all node densities. The byte delivery fraction decreases with the node density for all source-sink node velocities except velocity $\mathrm{v}=0$.
\end{abstract}

\section{KEYWORDS}

Sensor Networks, DSDV, Node Density

\section{INTRODUCTION}

The recent development in small embedded sensing devices and the wireless sensor network technology has provided opportunities for deploying sensor networks to a range of applications such as environmental monitoring, disaster management, tactical applications etc [1]. Main requirement for such application is that motes carrying onboard sensors should be physically small, low power consuming and include wireless radio. For data collection a straight forward solution is that each mote transmits its data to a centralized base station. However in such cases the energy requirement of each node would be large which reduces mote life and also there 
would be interference problem. Alternative approach for harvesting data from sensor fields uses mobile data collector such as robots which move in the sensor field to collect data and transmit the same to base station in real /non-real time [6].

In the present paper, the performance of destination sequenced distance vector (DSDV) protocol has been analyzed keeping in mind a sensor network scenario wherein all the nodes are static and two nodes are moving ( one of which is a data harvester from static nodes and other one acting as a sink).

Section 2 describes the related work and Section 3 is about the network scenario and all about the definition of simulation parameters and details of simulation experiment. Section 4 gives the results and discussion of the simulation. Section 5 concludes the work.

\section{Related Work}

\subsection{DESTINATION SEQUENCED DISTANCE-VECTOR ROUTING PROTOCOL (DSDV):}

DSDV is a table driven routing scheme for an ad hoc mobile networks based on the BellmanFord algorithm. It was developed by C. Perkins and P. Bhagwat in 1994 [2].The main contribution of this algorithm was to solve the routing loop problem. In DSDV each node maintains a route to every other node in the network and thus routing table is formed. Each entry in the routing table contains sequence numbers which are even if a link is present; else, an odd number is used. The number is generated by the destination, and the emitter needs to send out the next update with this number [5]. This number is used to distinguish stale routes from new ones and thus avoids the formation of loops. The routing table updates can be sent in two ways: a "full dump" or an incremental update. A full dump sends the full routing table to the neighbours and could span many packets whereas in an incremental update only those entries from the routing table are sent that have a metric change since the last update and it must fit in a packet. When the network is relatively stable, incremental updates are sent to avoid extra traffic and full dump are relatively infrequent. In a fast changing network, incremental packets can grow big so full dumps will be more frequent.

DSDV was one of the early algorithms available. It is quite suitable for creating ad hoc networks with small number of nodes. DSDV requires a regular update of its routing tables, which uses up battery power and a small amount of bandwidth even when the network is idle. Whenever the topology of the network changes, a new sequence number is necessary before the network reconverges; thus DSDV is not suitable for highly dynamic networks. 


\section{Network Scenario and Simulation Details}

The simulation scenario consists of tcl script that runs over TCP connections for various number of nodes $(20,40,60,80$ and of 100 nodes $)$ in an area of size of $1000 \times 1000 \mathrm{~m}^{2}$.Figure 1 shows the random node topology of the network. The simulation time is set to 150 seconds. TCP connection has been used to set communication between the source node and the sink node.

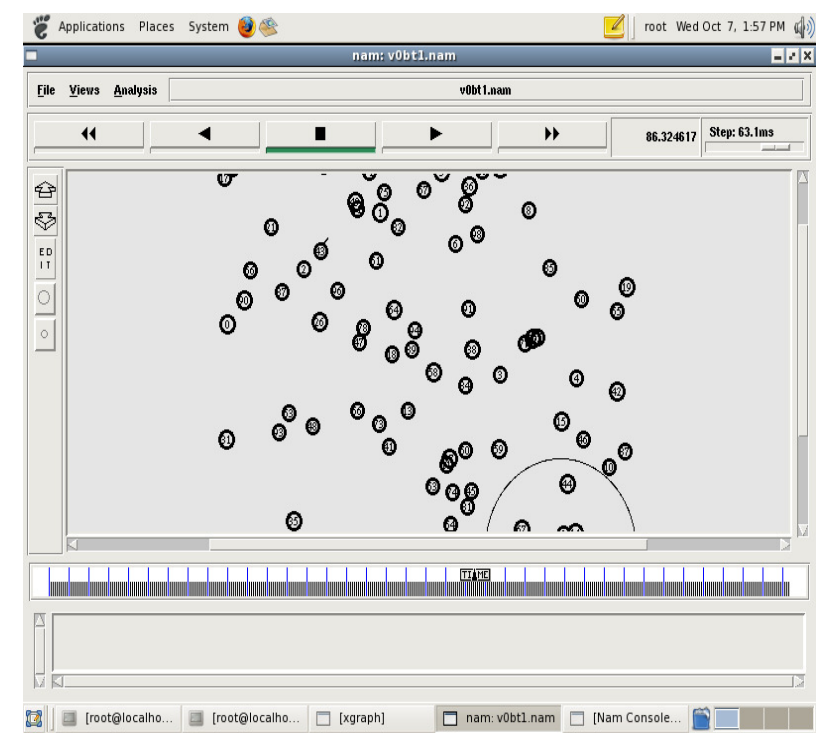

Figure 1: NAM Windows showing network topology for 100 nodes

The simulation is carried out in the network simulator NS-2 (version-2.31) over LINUX (FEDORA 8) environment [3] [4].The experiment has been performed for a set of 5 random network topologies having number of nodes $20,40,60,80$ and 100 respectively. In each topology all nodes are fixed except the source and sink nodes. For a given topology two nodes are selected to be source-sink nodes and the observations are made for five different velocities $0,10,20,40$ and $60 \mathrm{~m} / \mathrm{sec}$. The experiment was repeated by selecting another pairs of source-sink nodes chosen randomly. Five such observations were taken for each of the following three performance parameters and their averages are obtained to plot graphs:

1. Bytes Delivery Fraction: The ratio of the number of data sends (in bytes) successfully delivered to the destinations to those are generated by TCP agents/sources.

Thus, Bytes Delivery Fraction $=($ Received data $/$ Total sent data $)$

2. Average Number of Dropped Packets: This parameter is worth mentioning while taking the effectiveness of this routing protocol. These are the average number of dropped packets in simulation study of our scenario. 
3. Average Simulation End to End Delay: The delivery delay for the data is the interval between when it is generated at a sensor node and when it is collected by a sink [2009]. It does not depend on the time for collecting data from the network. These are the possible delays caused by buffering during route discovery latency, queuing at the interface queue, retransmission delays at the MAC and propagation and transfer times.

Simulation Parameters: The parameter values for simulation are given in table 1 .

\begin{tabular}{|l|l|}
\hline $\begin{array}{l}\text { Maximum simulation } \\
\text { time }\end{array}$ & 150 seconds \\
\hline Area size (Flat area) & $1000 \times 1000 \mathrm{~m}^{2}$ \\
\hline $\begin{array}{l}\text { Routing protocol } \\
\text { (proactive) }\end{array}$ & DSDV \\
\hline Propagation Model & $\begin{array}{l}\text { Two Ray Ground } \\
\text { Propagation }\end{array}$ \\
\hline MAC layers protocol & IEEE802.11 \\
\hline Node placement & $\begin{array}{l}\text { Static } \\
\text { Distribution Random }\end{array}$ \\
\hline Number of nodes & $20,40,60,80,100$ \\
\hline $\begin{array}{l}\text { Velocities of source and } \\
\text { sink nodes }\end{array}$ & $0,10,20,40,60 \mathrm{~m} / \mathrm{sec}$ \\
\hline
\end{tabular}

Table1. Parameter values for simulation

\section{Results and Discussion}

The average simulation end to end delay (as it is given in figure2) for static nodes scenario is generally higher than that in mobile source-sink case (an exception is attained at node density = 20 nodes $/ \mathrm{km}^{2}$. Moreover in static case from 20 nodes $/ \mathrm{km}^{2}$ to 40 nodes $/ \mathrm{km}^{2}$ the delay increases but for $40-80$ nodes $/ \mathrm{km}^{2}$ the delay is decreased which is just because more nodes are available in the network through which routing is possible. However for $80-100$ nodes $/ \mathrm{km}^{2}$, the delay is again increasing, which is caused by node density reaching very high. In the case of mobile sourcesink, the delay is almost constant at 0.12 seconds (except at v10 $=10 \mathrm{~m} / \mathrm{s}$ for 40 nodes $/ \mathrm{km}^{2}$ ). Again in this case the delay is much less (less than $50 \%$ than that for static case) because in these dynamic scenarios, the possibility of finding routes is more. 


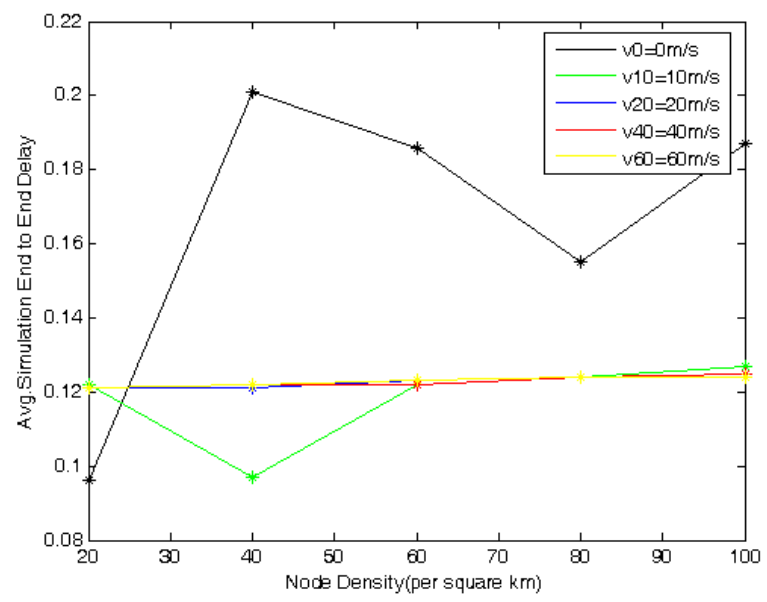

Figure2.Avg.simulation end2end delay vs. node density

The average number of dropped packets in static case is higher than that in dynamic source-sink scenario. This is because in later case once route is formed the possibility (link-breakage) is lesser as compared to the former case, where the probability of route formation is lower. Moreover as we see in figure3, in mobile source-sink case, the average number of dropped packets is of the vibrating nature at value 10 .

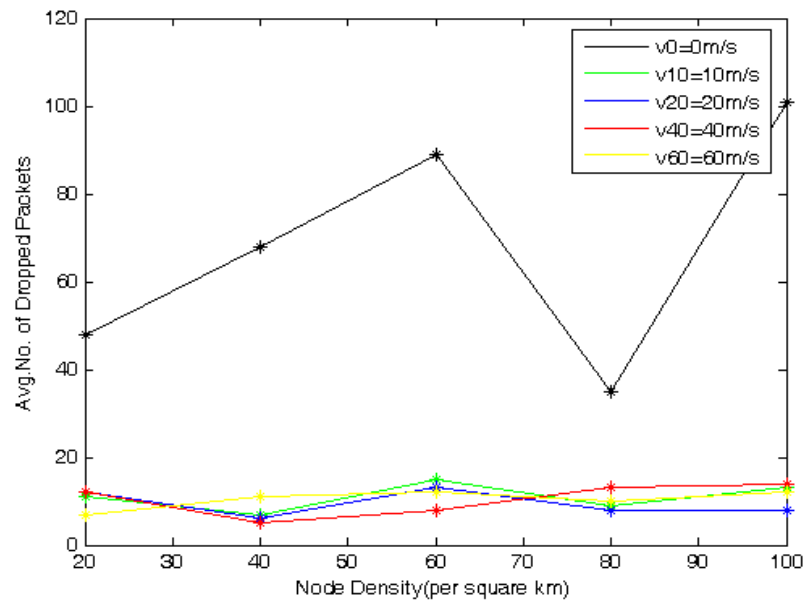

Figure3.Avg.no. of dropped packets vs. node density

As expected, the byte delivery fraction for moving source-sink case is higher than that for static case (which is evident from the graph for average number of dropped packets where it is less for the mobile source-sink case as compared to the static case). One more fact is evident from Figure 4 that the nature of graph for all cases (v0, v10, v20, v40 and v60) is decreasing when node density is increased. 


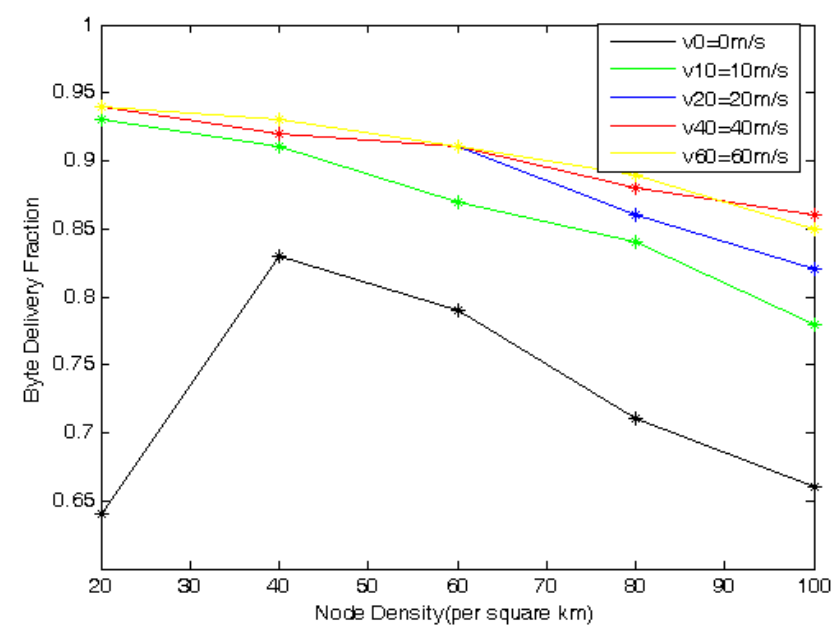

Figure4. Byte delivery fraction vs. node density

\section{Conclusion}

The end to end Delay for static node case is generally higher than those for mobile source-sink cases for all node densities. For later cases, it is almost constant. The average number of dropped packet for static node case is higher than those in dynamic cases. In dynamic scenarios, this parameter is around 10. The byte delivery fraction for dynamic source-sink cases is higher than that in static case. This parameter decreases with increasing node densities for all source-sink velocity cases. It may be mentioned that similar results have been reported for AODV, DSDV and I-DSDV protocols for mobile ad hoc networks with node densities 5 to 35 nodes $/ \mathrm{km}^{2}$ using Random Way Point Mobility Model [7]. Further work can be done to investigate performance of other manet protocols for the network scenario as considered in the present paper. 


\section{References}

[1] Hac, Anna, 2003. “Wireless Sensor Network Designs”, John, Wiley and Sons.

[2] Perkins., C. E., and P. Bhagwat., 1994. "Highly Dynamic Destination-Sequenced DistanceVector Routing (DSDV) for Mobile Computers", ACM, pp.234-244.

[3] The Network Simulator NS-2 homepage, http://www.isi.edu/nsnam/ns

[4]The Network Simulator NS-2 tutorial homepage

http://www.isi.edu/nsnam/ns/tutorial/index.html

[5] Royer, E.M., and C. K. Toh. 1999."A review of current routing protocols for adhoc mobile wireless networks", IEEE Personal Communications, (April).

[6] Rao, J. and S.Biswas, 2008. "Data Harvesting In Sensor Networks Using Mobile Sinks", IEEE Wireless Communication, pp.1536-1284.

[7] Rahman, A. H. A., Zuriati, A. Zukarnain. 2009. "Performance Comparison of AODV,DSDV and I-DSDV Routing Protocols in Mobile Ad Hoc Networks",European Journal of Scientific Research, ISSN 1450-216X. Vol.31 No. 4, pp.566-576. 